\title{
The Effect of Contextualizing and Decontextualizing Techniques on Lexical- oriented Knowledge of Persian EFL Language Learners
}

\author{
Kamal Heidari Soureshjani \\ Young Researchers Club Member, Islamic Azad University, Shahrekord Branch, Shahrekord, Iran \\ Email: k_tefl_h@yahoo.com
}

\begin{abstract}
Taking a pretest posttest control group design, the present study served as an attempt to compare the effects of two vocabulary teaching techniques (contextualization and decontextualization techniques) on the elementary Iranian EFL learners. To achieve such a purpose, 190 male and female language learners were randomly selected and assigned into two groups, a control group and an experimental group. In the control group, the new English words were presented in word lists associated with their Persian meanings and students were supposed to memorize them (a decontextualizing technique). In the other group, the new English words were presented in model sentences (a contextualizing technique). The data were collected using two reliable and valid tests as pretest and posttest: Each of them consisted of 15 equivalent multiple-choice items of vocabulary. The result of the study revealed significant differences between the two groups of the study. The experimental group outperformed those in the control group quite significantly on vocabulary memorization test. The experimental group also had a better performance on a sentence-making test than the control group though the differences were not significant.
\end{abstract}

Index Terms - contextualizing, de-contextualizing, rote memorization, sentence-making technique

\section{INTRODUCTION}

It is a truism and has also been repeatedly mentioned by different researchers that assessing vocabulary is necessary. They support this claim by arguing that words are the basic building blocks of language and it is the units of meaning from which larger structures such as sentences, paragraphs and whole texts are formed (Read, 2000; Schmitt, 2000). Considering the crucial role of vocabulary learning in second or foreign language learning contexts, one can makes aware of the importance of vocabulary teaching as well. In the past, vocabulary teaching and learning were often given little priority in second language programs, but recently there has been a renewed interest in the nature of vocabulary and its role in learning and teaching (Richards \& Renandya, 2002). Furthermore, some studies have shown lexical problems of language learners indicating that lexical problems frequently interfere with communication. In fact, communication breaks down when people do not use the right words (Allen, 1983). Therefore, there is an increased interest in vocabulary as a component of every language.

There have been proposed different techniques for teaching vocabulary items in methodology textbooks. Generally speaking, there are three approaches to vocabulary teaching: incidental, or indirect learning (learning words as a byproduct of doing other things such as reading or listening), explicit or direct learning (diagnosing the words learners need to know, presenting them to the learners and elaborating on their word knowledge) and independent strategy development (practicing by guessing meaning of the words from content and training learners to use dictionaries) (Hunt \& Beglar, 2000). As to the first two approaches to vocabulary teaching, a number of techniques can be employed. One is to use contextualizing techniques such as memorizing word lists or using flashcards. While adopting the indirect approach, one way is using contextualizing techniques such as reading and listening practice and speaking and writing practice (oxford \& Crookall, 1990).

Regarding all these studies and findings, however, there is a controversial issue based on which whether the direct approach is superior to the indirect approach. Most of the studies in this regard have shown mixed results. Read (2004) for instance asserted that although learners certainly acquire word knowledge incidentally while engaging in various language learning activities, more direct and systematic studies of vocabulary is required.

\section{BACKGROUND TO THE STUDY}

Assorted studies have dealt with the effects of the direct and indirect approaches of vocabulary teaching on learning vocabulary some of which are presented in this section. Lawson \& Hagen (1996) for example investigated the behavior of the university students attempting to learn the meanings of new Italian words using a thinking aloud procedure. The 
procedures involved repetition of the new words and their meanings. There was little use of the physical or grammatical features of words, or elaborative acquisition procedures. This lack of acquisition between use of content and recall of word meaning is contrasted with the stress placed on content by some researchers. Even when the subjects used the cues in the sentences to generate possible meanings for the target words, it didn't help them establish representations for the meanings of the words.

Khuwaileh (1995) also investigated the effect of contextualization techniques on vocabulary learning of the intermediate-level Jordanian university students $(\mathrm{N}=40)$. There were two lists, each containing twenty new words. List one was presented with English meaning and discussed in Arabic, the words of list two were in a text for silent reading with vocabulary questions. After 12 weeks, the students were tested on both groups of words. The results showed a clear advantage of contextualization for comprehension, learning and recall.

It is often heard that most words are learned from context and it is a very useful and productive way to learn words, however the usefulness of this method of vocabulary learning for all learners at different levels of proficiency is questionable. Waring (1955), for example, argued that beginners need a basic vocabulary before they can even start to learn from contexts as they have insufficient knowledge and the tent is too dense with unknown and known words. In fact, they do not also have enough knowledge of grammar and cannot make use of grammatical contextual clues to guess meaning from the context. He then suggested that guessing from context be left to a later stage when the learners have enough knowledge base.

Language learning process is not complete without vocabulary. It is impossible to learn a language without vocabulary. The important role of vocabulary has been emphasized in all different methods in language teaching. Rivers (1981) stated that vocabulary cannot be taught. It can be presented, explained and included in all kinds of activities however it must be learned by the individuals. She then goes on by saying that language teachers must arouse interest in words and a certain excitement in personal development in this area. She finally suggested that language teachers must help their students by giving ideas on how to learn vocabulary.

On the whole, with regard to the afore-cited studies, most of studies support the point that decontextualizing vocabulary learning techniques are superior to contextualized techniques. Just one study (Khuwaileh, 1995) supported the use of contextualizing techniques for vocabulary learning. In addition, some other studies like Qian's (1996) one that made a comparison between the learning of second language words in lists and contexts, failed to show any significant effects for one method over the other. These different results highlight the necessity of conducting more studies in different contexts to get more reliable findings.

Although vocabulary has been the subject of many studies, few researchers have revealed the effective techniques of vocabulary teaching. Therefore, it is very important to find the most effective technique for vocabulary teaching. All experienced language teachers confirm the important role of words and know that the lack of them leads to feeling of insecurity. Besides, teachers use different vocabulary teaching techniques such as lists, synonyms, antonyms, etc.

The present study aimed to show the effects of two vocabulary teaching techniques, that is, rote memorization of word lists (a de-contextualizing technique) and sentence-making practice , (a contextualizing technique) on learning English vocabulary as a foreign language. Totally, the study tries to cast light on the following research questions:

1. Is there any difference between the performance of the students on vocabulary memorization test and sentencemarking test?

2. Is there any significance difference between students' vocabulary learning via contextualizing and decontextualizing techniques?

The study findings can add to the body of knowledge as to finding the most effective vocabulary teaching methods and making language learners be more efficient in their learning.

\section{METHODOLOGY}

\section{A. Participants}

Altogether 90 male and female language learners were randomly selected (from among almost 190 learners) to take part in the study. They aged from 17 to 23 and all were taking beginning courses in two of language institutes in Shiraz, Iran. Having confirmed their homogeneity, they were randomly assigned to two groups, an experimental group and a control group, each consisting of 45 students. The experimental group received the treatment that is, teaching vocabulary items contextually. And the control group received vocabulary items through word lists in English along with their translations in Persian.

\section{B. Materials}

In order to conduct the study two tests were constructed: a pretest and a posttest. These tests were standard and valid tests based on the book New Generation by Colin Granger and Digby Beaumount (1987) which has been used at the institutes for several years. The pretest was a vocabulary test prepared by the researcher involving 30 vocabulary items all in the multiple-choice format and then did a pilot study on a smaller group $(\mathrm{N}=25)$. During the pilot study some of the items were found out to be changed or completely omitted and finally the number of items reduced to 20 valid and standard items. The reliability of the pre-test and post-test were calculated to be 0.81 and 0.77 respectively using KR-21 
formula. The reliability of the sentence- making test was also computed through the same formula and it turned out to be 0.77 which is an acceptable index of reliability.

\section{Data Analysis}

As it was already mentioned, the participants were randomly assigned into two groups. One group served as the experimental group in which students worked on the vocabulary items through sentence-making practice both in written and spoken form (a contextualization technique). The point needs to be raised here is that this method is the most common way of teaching vocabulary in the institutes. The other group served as the control group in which students tried to learn the words through rote memorization of word lists. The researcher asked the students to elicit the meaning of the words appearing in the wordlists both in English and Persian.

The vocabulary pretest was administered to both experimental group and control group. The time allotment for the test was forty minutes. The correct answer to each item received one point, and there was no penalty for wrong responses. The whole research project took place in almost 60 days and the students were taught five units of the book. During the project, the new words of each unit were presented to the students through two methods of contextualization and de-contextualization. In the experimental group the words were used in some model sentences. In control group, the new words were presented through definitions and synonyms. At the end of the sessions, vocabulary development of the students in both groups was tested using the posttest. On the basis of these tests the efficacy of two methods of vocabulary teaching was then determined.

\section{RESULTS}

In order to begin the study and in order to make sure about the homogeneity of the students, the researcher administered a pretest to both control and experimental groups unexpectedly in the second session. Table 1 presents the descriptive statistics of this pretest.

TABLE 1.

TABLE 1.
\begin{tabular}{|c|c|c|c|c|}
\hline DESCRIPTIVE STATISTICS OF THE PRETEST \\
\hline No & Min & Max & Mean & SD \\
\hline 90 & 5 & 27 & 19.22 & 8.30 \\
\hline
\end{tabular}

Tables 2 and 3 also represent the descriptive statistics for the two tests given to the students at the beginning and end of the course.

TABLE 2.

DESCRIPTIVE STATISTICS OF THE ROTE MEMORIZATION TEST
\begin{tabular}{|c|c|c|c|c|}
\hline No & Min & Max & Mean & SD \\
\hline 90 & 16 & 58 & 35.50 & 12.30 \\
\hline
\end{tabular}

And finally, based on Table 4, Beta values indicates that one standard deviation unit change in the score for autonomy will result in 0.52 unit of change in GPA. However, one standard deviation unit change in the score for motivation will result in 0.34 unit of change in GPA. Thus, on the whole, it can be inferred that autonomy scores are better predictors of GPA than motivation:

TABLE 3.

DESCRIPTIVE STATISTICS OF THE SENTENCE MAKING TEST

\begin{tabular}{|c|c|c|c|c|}
\hline No & Min & Max & Mean & SD \\
\hline 90 & 4 & 18 & 11.50 & 3.40 \\
\hline
\end{tabular}

Then in order to make a comparison between the two groups to see if there is any statistically significant difference between the two groups, the obtained raw scores were subjected to two separate independent t-tests. As table 4 indicates, the experimental group had a remarkably better performance than the control group on the rote memorization test.

TABLE 4.

INDEPENDENT T-TEST COMPARING THE PERFORMANCE OF THE TWO GROUPS ON ROTE MEMORIZATION TEST

\begin{tabular}{|c|c|c|c|c|c|c|}
\hline Variable & No & Mean & SD & S.E. of mean & DF & 2 -tail sig. \\
\hline EXP & 15 & 38.94 & 12.70 & 2.750 & 35 & 0.02 \\
\hline Cont & 15 & 31.95 & 10.34 & 2.176 & & \\
\hline
\end{tabular}

Table 5 also reveals that with regard to sentence-making test the two groups performed very similarly however, the experimental group was minimally better than control group performance.

TABLE 5.

INDEPENDENT T-TEST COMPARING THE PERFORMANCE OF THE TWO GROUPS ON THE SENTENCE MAKING TEST

\begin{tabular}{|c|c|c|c|c|c|c|}
\hline Variable & No & Mean & SD & S.E. of mean & DF & 2-tail sig. \\
\hline EXP & 45 & 11.65 & 3.30 & .736 & 45 & 0.95 \\
\hline Cont & 45 & 11.55 & 3.50 & .724 & & \\
\hline
\end{tabular}


Finally with the end of the course, the posttest was administered to both experimented and control groups. The descriptive statistics of this test are shown in table 6.

TABLE 6.

DESCRIPTIVE STATISTICS OF THE POST-TEST

\begin{tabular}{|c|c|c|c|c|}
\hline No & Min & Max & Mean & SD \\
\hline 90 & 8 & 22 & 32.05 & 7.22 \\
\hline
\end{tabular}

Then in order to see whether the treatment given to the experimental group had caused any significant change in their performance; another independent t-test was run. Table 7 well shows that the experimental group outperformed the control group but not significantly.

TABLE 7.

INDEPENDENT T-TEST COMPARING THE PERFORMANCE OF THE TWO GROUPS ON THE SENTENCE MAKING TEST

\begin{tabular}{|c|c|c|c|c|c|c|}
\hline Variable & No & Mean & SD & S.E. of mean & DF & 2-tail sig. \\
\hline EXP & 45 & 33.30 & 6.50 & 1.370 & 46 & 0.27 \\
\hline Cont & 45 & 30.86 & 7.90 & 1.550 & & \\
\hline
\end{tabular}

\section{DISCUSSION AND CONCLUSION}

The main purpose of the current study was to ascertain the probable effects of decontextualizing and contextualizing teaching methods on the performance of Iranian EFL elementary language learners on lexical tests. To put another way, the study tried to find which one of these two methods is more effective, appropriate, and useful for elementary-level language learners in their lexical performances.

The results of the study which were obtained from the analysis of data pertaining to the pretest and posttest made clear that although the differences between the means of the experimental and control group was not statistically significant, the treatment given to the experimental group had affected this group performance to some extent.

The results of the sentence-making test also revealed that the experimental group did better on this test as compared to the control group, though this difference was again marginal. The significance of the results doubles when one pays attention to the fact that the students in the control group had a lot of sentence-making practice both in written and spoken form in the class while the students in the experimental group did not have such practice. It can be inferred that good recall of the meaning of the vocabulary items might have helped students in the experimental group to make appropriate sentence and that the students in the control group did not perform as well as they were expected to on this test simply because they could not recall the meanings of the words which is a basic requirement for making sentences using such words. Another reason for the low performance of the subjects in the control group is that these students usually copy sentences from dictionaries when they are asked to make sentences based on vocabulary items. In fact, they do not actually make sentences to have real practice in sentence-making.

The results of this study support that of Qian (1996) who suggests that for some language learners de-contextualized second language vocabulary learning with feedback is more effective than contextualized vocabulary learning without feedback. He also challenges the assumption that contextualized vocabulary learning always leads to better recall. Another support for the results of the study comes from a research project undertaken by Laufer and Shumueli (1997), based on which words glossed in first language were shown to be always better retained than those glossed in second language. The results of this study can further be supported by Waring (1995) who has questioned the appropriateness of contextualized method of vocabulary learning for all learners. He concludes that beginners requires a body of basic vocabulary items before they can start learning from context since they do not have enough knowledge of the words and the text is too difficult for them.

The appropriateness of the use of word lists, as employed in the experimental group of this study, is also recommended by Meara (1995, reported in Critchley, 1998) who maintains that presenting vocabulary items in list form is on efficient study method in which students can learn many words in a short time. The results of this study suggest that presenting vocabulary items in word lists rather than in context is better for beginning learners. Consequently, attempts should be made to provide beginners with English textbooks in which English words associated with their Persian meaning are presented.

One of the most notable implications which can be inferred from this study is that since a good knowledge of vocabulary has a great effect on the learners' improvement of other aspects of language such as reading comprehension, listening comprehension, speaking and writing, specially at beginning levels, more attention should be paid to choosing and implementing appropriate vocabulary teaching learning techniques in language classes. Based on the findings of this study, it can be concluded that rote memorization of word lists as a de-contextualizing or direct technique of vocabulary teaching/learning is more useful than sentence-making practice as a contextualizing or indirect technique, and it can play on important role in teaching vocabulary items particularly for learners at the beginning level of language instruction. 


\section{APPENDIX A PRETEST OF VOCABULARY}

1. I can ----- in water.
a) drive
b) cook
c) climb
d) swim

2. Kate's father can -----a car.
a) work
b) drive
c) study
d) run

3. There is a hole. You should..... .
a) eat
b) jump
c) write
d) read

4. I am going to France.

Have you got a ---- ?
a) magazine
b) notebook
c) passport
d) piano
5. It is very dark. Have you get a ---- ?
a) ball
b) torch
c) newspaper
d) ladder

6. There is a high ----. You should climb it.
a) mountain
b) zebra
c) sack
d) tap

7. You can ski, because there is a deep -----
a) bucket
b) snow
c)magazine
d) watch

8. He was killed in a car. ......
a) accident
b) net
c) light
d) magazine

9. There is a lot of ----- at 8 o'clock in the morning. There are many cars in the street.
a) kite
b) cinema
c) restaurant
d) traffic

10. A. ---- is a large strong car for carrying things.
a) lorry
b) gun
c) motorbike
d) taxi

11. There are four ------- in a year.
a) days
b) months
c)hours
d)seasons

12. Which one can be used for swimming?
a) bat
b) goggles
c) gloves
d) towel

13. There is no cloud in the sky. It is ------------
a) snowy
b) clear
c) rainy
d) windy

14. It is too far to the post office. Let's -------- a taxi.
a) go
b) take
c) make
d) run

15. I can not talk to you now. I have many things to do. I am really
a) relaxed
b) old
c) busy
d) glad

\section{APPENDIX B. POST-TEST OF VOCABULARY}

1. What is your father's job? He mends cars. He is a -----.
a) journalist
b) pop singer
c) waiter
d) mechanic
2. That man carrying the letters is a-----.
a) postman
b) teacher
c) singer
d) footballer
3. Harry can -------- the mountain.
a) climb
b) put
c) wash
d) ride

4. My brother can -------- when there is snow.
a) open
b) wash
c) ski
d) cook

5. There is a snack. You should -------.
a) run
b) clean
c) swim
d) do

6. Bert can row a---------- in the sea.
a) cup
b) boat
c) coat
d) poster

7. Allen's father can fly a ----.
a) bicycle
b) car
c) house
d) plane

8. There is a --------. You can swim in it.
a) lake
b) park
c ) road
d) mountain

9. It is cold. Have you get a --------?

I want to make fire.
a) key
b) map
c) lighter
d) desk
10. I have got a --------. Aren't you hungry?
a) ring
b) sandwich
c) drink
d) passport
11. Please
- a circle on the board.
a) make
b) jump
c) take
d) draw
12. There
b) walk
c) ski
d) jump 
13. Can you bring me a ------- of water for me?
a) can
b) battle
c)glass
d) tube
14. I think Ali is late. I should him.
a) call
b) shout
c) go
d) see
15. The sky is clear. I see many --------- in the sky.
a) moons
b) clouds
c) stars
d) lights

\section{APPENDIX C. SAMPLE VOCABULARY ITEMS PRESENTED THROUGH CONTEXTS}

\section{Lesson One}

1. Monkeys can climb the trees well. They go up the trees very fast.

2. A: Can you drive a car?

B: No, I can't. But my father can drive. He is a taxi driver.

3. Look! The children are very happy. They are jumping up and down.

4. He is late for the bus. He is running very fast to the bus-stop.

5. Your hands are dirty. You should wash them before lunch.

6. Allen's mother is in the kitchen. She is cooking pizza for dinner.

7. Bob's father was not a good driver. Last year, he was killed in a car accident.

8. When the cars come to crossroads, they should stop to let the other cars pass.

\section{Lesson Two}

1. If you want to keep the food cold, you should put them in the refrigerator.

2. There is a net in the garden and the children are playing volleyball.

3. Look! There are two rackets and a net here. Let's go to play tennis in the yard.

4. I want to play tennis with my friend. But I can't find my racket.

5. Michael and Allen played chess with each. At the end, Michael checkmated Allen's king.

6. Harry is swimming across the river. He is swimming on his back.

7. A child felt into the river. Her father dived into the water to serve her.

8. What Kind of car have you got?

\section{REFERENCES}

[1] Allen, V. F. (1983). Techniques in teaching vocabulary. New York: Oxford University Press.

[2] Critchley, M. P. (1988). Reading to learn: Pedagogical implications of vocabulary research. The Language Teacher, 22(12), 1519.

[3] Granger, C. \& Beaumount, D. (1987). New generation. Cambridge: Cambridge University Press.

[4] Hunt, A., \& Beglar, D. (2000). Current research and practice in teaching vocabulary. Cambridge: Cambridge University Press.

[5] Khuwaileh, A. A. (1995). Words and context in EFL. Grazer Linguistics Student, 44, 27-36.

[6] Laufer, B., \& Shmueli, K. (1997). Memorizing new words: Does teaching have anything to do with it? RELIC JOURNAL, 28(1), 89-106.

[7] Lawson, M. J., \& Hogben, D. (1996). The vocabulary- learning strategies of foreign- language students. Language learning, 46 (1), 101-135.

[8] Oxford, R., \& Crookall, D. (1990). Vocabulary learning: A critical analysis of techniques. TESL Canada Journal, 7(2), 9-30.

[9] Qian, D. D. (1996). ESL vocabulary acquisition: Contextualization and decontextualization. The Canadian Modern Language Review, 53(1), 120- 142.

[10] Read, J. (2000). Assessing vocabulary. Oxford: Oxford University Press.

[11] Read, J. (2004). Research in teaching vocabulary. Annual Review of Applied Linguistics, 24, 146-161.

[12] Richards, J. G., \& Renandya, W. A. (2002). Methodology in language teaching: An anthology of current practice. Cambridge: Cambridge University Press.

[13] Rivers, W. M. (1981). Foreign language skills. Chicago: University of Chicago Press.

[14] Schmitt, N. (2000). Vocabulary in language teaching. Cambridge: Cambridge University Press.

[15] Waring, R. (1995). Second language vocabulary acquisition: Linguistic context and vocabulary task design. Retrieved December 10, 2008 from http: www. harenet. ne.ip/ warign/papers/BC. html.

Kamal Heidari Soureshjani holds an M.A. in TEFL from Shiraz University. He has published papers in different journals including TPLS. He has also taught English courses and IELTS at different institutes in Shiraz and is presently the academic member of Islamic Azad University, Shahrekord Branch. 